\title{
Coupling Strength Can Control the Polarization Twist of a Plasmonic Antenna
}

\author{
Banafsheh Abasahl, Shourya Dutta-Gupta, Christian Santschi, and Olivier J. F. Martin* \\ Nanophotonics and Metrology Laboratory (NAM), Swiss Federal Institute of Technology Lausanne (EPFL), 1015 Lausanne, \\ Switzerland
}

ABSTRACT: The far-field polarization of the optical response of a plasmonic antenna can be tuned by subtly engineering of its geometry. In this paper, we develop design rules for nano antennas which enable the generation of circular polarized light via the excitation of circular plasmonic modes in the structure. Two initially orthogonal plasmonic modes are coupled in such a way that a rotational current is excited in the structure. Modifying this coupling strength from a weak to a strong regime controls the helicity of the scattered field. Finally, we introduce an original sensing approach that relies on the rotation of the incident polarization and demonstrates a sensitivity of $0.23 \mathrm{deg} \cdot \mathrm{nm}^{-1}$ or $33 \mathrm{deg} \cdot \mathrm{RIU}^{-1}$, related to changes of mechanical dimensions and the refractive index, respectively.

KEYWORDS: Plasmonics, antennas, coupling strength, polarization, sensors
T $\mathrm{n}$ the past few decades, localized plasmon resonances supported by metallic nanostructures have attracted significant attention thanks to their applications in a variety of fields, such as biosensing, ${ }^{1}$ photovoltaics, ${ }^{2}$ and optoelectronics. ${ }^{3}$ It is now well-known that, with appropriate tuning of a plasmonic nanostructure, it is possible to engineer both its near and far-field response. ${ }^{4-8}$ In the optical regime, plasmonic nanostructures react similarly to antennas in the sense that incident light can be collected and stored in the near-field, and conversely, energy stored in the near-field can be radiated into the far-field. The far-field emission pattern of planar plasmonic structures is determined by the near-field distribution. As a consequence, it is possible by engineering the near-field of a plasmonic nanostructure to design its response in a way such that a linearly polarized excitation results in a circular polarized (CP) response. ${ }^{9-18}$ Recently, nanostructures supporting not only one single plasmon mode but also multiple interacting plasmonic modes have been developed. ${ }^{19-23}$ The interaction between such plasmonic modes allows energy transfer between the particular modes within the corresponding wavelength range, and consequently, different modes can indirectly be excited through their near-field. In line with the observed asymmetric scattering spectra, these modes are named Fano resonances. ${ }^{24}$ Potential applications of such structures exhibiting Fano line shapes include sensing, energy storage, and spectroscopy enhancement. ${ }^{25-28}$ Most of those applications rely on the modulation of the intensity of the scattered light induced by the interaction of the plasmonic modes over different spectral regions. It has already been shown that light polarization can be altered using plasmonic nano antennas ${ }^{29-31}$ or plasmonic metamaterials. ${ }^{32-34}$ In this paper, we show that the polarization of the scattered light can be controlled by introducing a perturbation into a system with initially orthogonal plasmonic modes. Furthermore, we demonstrate that such structures can be used for the generation of CP light from an incident linearly polarized plane wave. Finally, we show, as an application example, that these structures are very sensitive to changes in dimensions and can therefore be used as extremely sensitive sensors for mechanical displacements or changes in refractive index.

Metallic nanoparticles, with dimensions much smaller than the incident wavelength behave like nanoantennas, hence, exhibit a dipolar charge distribution when they are excited. In this paper, we consider a plasmonic structure consisting of two perpendicularly arranged elliptical nanostructures (Figure 1a inset), each having a major semiaxis $a=60 \mathrm{~nm}$. In the center of the intersection area resides an elliptical or circular cavity for the asymmetric or symmetric configuration, respectively. The reported charge distributions and scattering cross sections are simulated using the surface integral method, ${ }^{35}$ and the excitation is a normal incident $x$-polarized plane wave (propagating along the $-z$-direction, Figure 1a), unless stated otherwise. In the symmetric configuration, under this illumination condition, resonator (2) along the $y$-direction cannot be excited as its resonance mode is perpendicular to the excitation polarization. In other words, antenna (2) is dark for an excitation along the $x$-direction, since in this symmetric configuration, the two modes of the system are degenerated and orthogonal, and, hence, an energy exchange cannot take place. Let us recall that the required condition for the generation of CP light is the superposition of two beams with perpendicular polarizations of equal intensity and a $\pi / 2$ phase difference. In the symmetric configuration (Figure 1a), no CP can be generated since no interaction occurs between the $x$ - and $y$-resonators. When a nonsymmetrical perturbation

Received: August 5, 2013

Revised: August 27, 2013

Published: August 29, 2013 

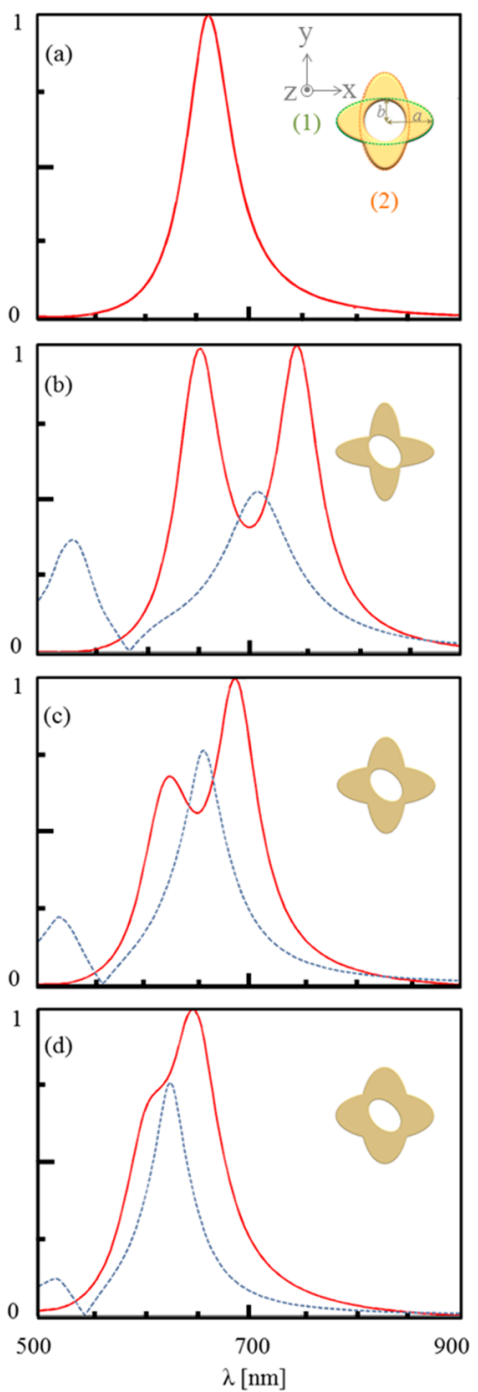

Figure 1. Normalized scattering cross section (red solid lines) and magnitude of circular polarization $\left|\alpha_{c}\right|$ (blue dashed lines) for the four cases: (a) unperturbed double dipole antenna, perturbed structures in three different coupling regimes: (b) strong, (c) intermediate, and (d) weak coupling. The inset in each panel shows the corresponding schematic structure. In all cases, the excitation is polarized along the $x$ axis.

in the form of an elliptical cavity is introduced in the system, as shown schematically in the insets of Figure $1 b-d$, its symmetry is broken, the modes are no longer orthogonal, and consequently, energy can transfer between them. In contrary to previously reported structures, ${ }^{36-43}$ here both antennas are electrically connected. They can be engineered in such a way that circular currents are induced, allowing energy transfer between $x$ - and $y$-resonators and, consequently, a coupling of the two modes takes place. To achieve equal coupling between both modes, a $45^{\circ}$ oriented elliptical cavity with major and minor semiaxes of 25 and $15 \mathrm{~nm}$ is placed in the overlap region (insets in Figure $1 \mathrm{~b}-\mathrm{d}$ ).

The parameter $\alpha_{\mathrm{c}}$ is introduced as the degree of CP:

$$
\alpha_{\mathrm{c}}=\frac{\left|C_{\text {right }}\right|-\left|C_{\text {left }}\right|}{\left|C_{\text {right }}\right|+\left|C_{\text {left }}\right|}
$$

In this formula, $C_{\text {left }}$ and $C_{\text {right }}$ are the coefficients of the electric field decomposition into the two orthogonal left- and right-handed circular polarizations. $\alpha_{\mathrm{c}}=+1$ and $\alpha_{\mathrm{c}}=-1$ correspond to pure right-handed or left-handed CP, respectively, while linear polarization results into $\alpha_{\mathrm{c}}=0$.

Using this approach, we investigate in Figure 1 the generation of CP for four double-antenna structures with different dimensions exhibiting no, strong, moderate, or weak coupling. To use a realistic geometry for fabrication at the nanoscale, each antenna is chosen to have an elliptic shape. ${ }^{44}$ Figure 1a shows the normalized far-field scattering spectrum for a double antenna in the symmetric configuration. The length of the antenna minor semiaxis is $b=20 \mathrm{~nm}$, and the circular hole has a radius of $25 \mathrm{~nm}$. The scattering cross-section exhibits a single maximum located at $\lambda=661 \mathrm{~nm}$ (Figure 1a), as expected for the symmetric configuration where no energy is coupled to the $y$-mode. Compared to a similar single-elliptical antenna with hole, the resonance of this structure is $30 \mathrm{~nm}$ red-shifted, which indicates that, although the perpendicular dipole is not excited, its presence loads the antenna. ${ }^{45}$ Imposing a small perturbation to the system, as shown in Figure $1 \mathrm{~d}$, results in a marginal change of the scattering cross section, confirming the weak coupling of the two original modes. On the contrary, in the case of a strong perturbation, the modes of the unperturbed system evolve into new resonance peaks, as a result of strong coupling, Figure $1 \mathrm{~b}$. For the three cases studied here, the dimensions of the cavity and the major axis of the antenna ellipse are kept constant, but its minor axis $b$ is varied. The width of the antenna ellipse provides thus an appropriate parameter to control the impact of the perturbation on the system, namely, the strength of the coupling between both modes.

As mentioned, the introduction of an asymmetric perturbation in the form of an elliptical cavity splits the scattering peak of the original antenna into a symmetric line shape containing two peaks located at $\lambda=654$ and $\lambda=745 \mathrm{~nm}$ (Figure $1 \mathrm{~b}$ ), which clearly reveals a coupling between the two perpendicular antennas [(1) and (2) in the inset in Figure 1a]. The values of $\left|\alpha_{c}\right|$ for different wavelengths calculated along the $z$-axis at a distance of $50 \mu \mathrm{m}$ from the structure are shown in Figure 1 (dashed lines). At both peak positions the value of $\left|\alpha_{c}\right|$ is considerably lower than at the wavelength of the dip, which indicates that the polarization is more linear at the peaks than at the wavelengths close to the dip, which shows that generation of circular polarization occurs more efficiently close to the dip position. The maximum of $\left|\alpha_{c}\right|$ is close to the dip of the scattering cross section where the polarization contains a strong circular component.

To have a better insight into the underlying mechanisms, the time variations of the surface charge distribution over a single period corresponding to the two peak wavelengths of $\lambda=654$ and $\lambda=745 \mathrm{~nm}$ and the wavelength corresponding to the maximum of $\left|\alpha_{c}\right|$ (viz. $\lambda=701 \mathrm{~nm}$ ) are presented in Figure $2 \mathrm{a}-$ c. The charge distribution at $\lambda=654 \mathrm{~nm}$ shows a dipolar behavior along the major axis of the elliptical hole. The same type of distribution can be seen at $\lambda=745 \mathrm{~nm}$; although, in contrast to $\lambda=654 \mathrm{~nm}$, this dipole is now oriented along the minor axis of the elliptical hole. At $\lambda=701 \mathrm{~nm}$, over one period, the charges travel in an elliptical path, elongated in the $y$-direction. At these three wavelengths, the $y$-oriented antenna (2), which is dark in the symmetric case for this particular excitation, can be excited and, consequently, radiates.

In the intermediate coupling regime, the minor semiaxis $b$ of the antennas has been increased from 20 to $25 \mathrm{~nm}$, while keeping the same cavity as in Figure $1 \mathrm{~b}$. For this configuration, the far-field spectrum and $\left|\alpha_{c}\right|$ are shown in Figure 1c. The 


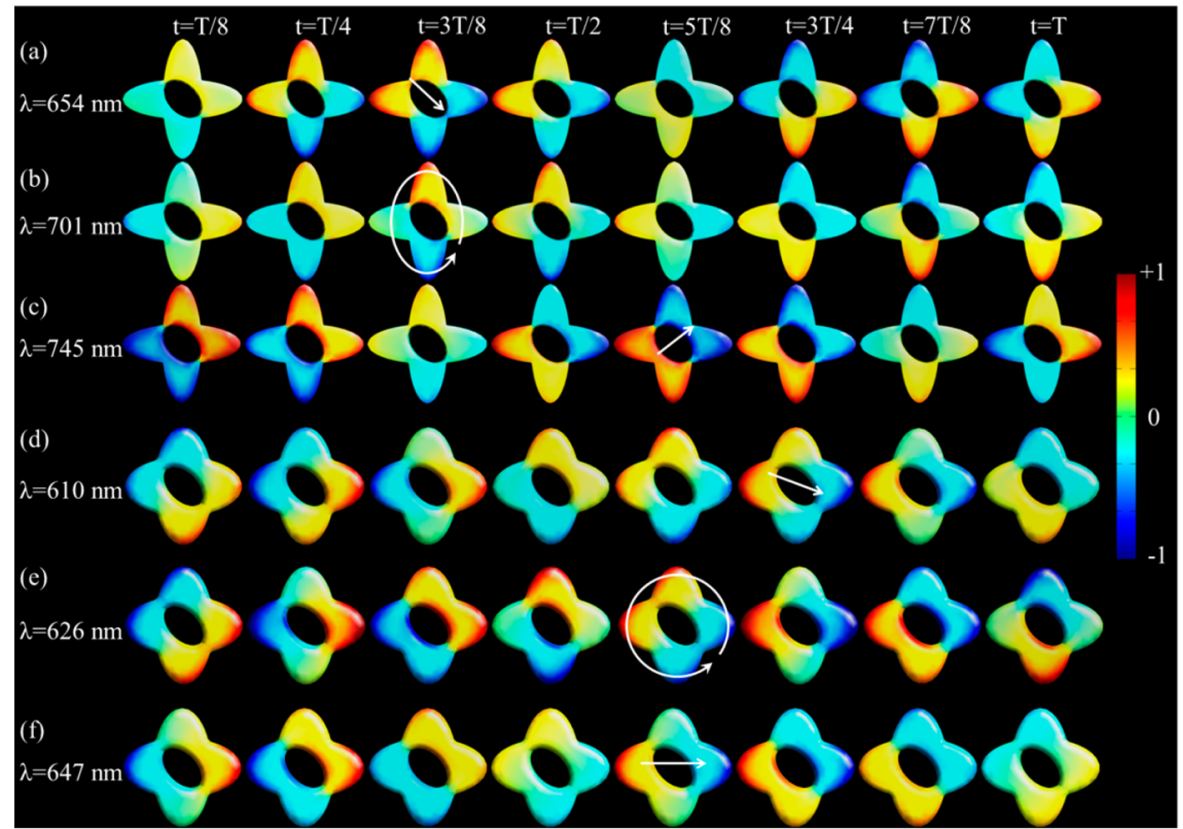

Figure 2. Time evolution of the charge distribution in the antenna in the strong $(a-c)$ and weak $(d-f)$ coupling regimes over one period $T$. The structures are excited with a polarization along the $x$-axis and the indicated wavelengths. The white arrows indicate the resulting polarization direction in the near-field, as well as in the far-field.
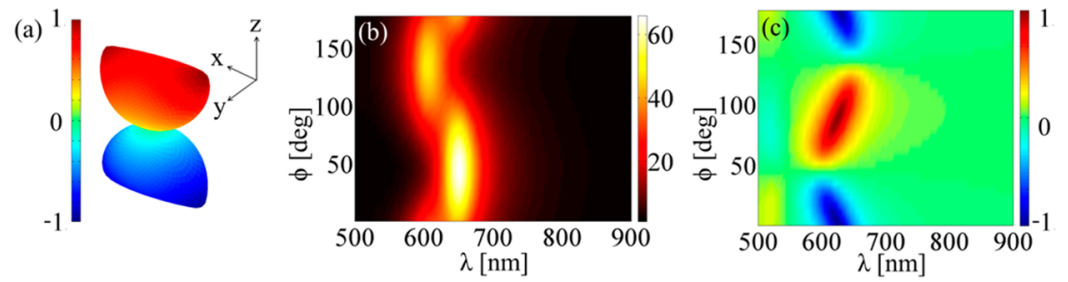

Figure 3. (a) Far-field CP angular distribution at the splitting point, $\lambda=626 \mathrm{~nm}$, for the structure in the weak coupling regime (inset in Figure 1d). (b) Scattering cross section and (c) the magnitude of $\alpha_{\mathcal{c}}$ as a function of the polarization angle $\varphi$ and the illumination wavelength.

current spectrum exhibits an asymmetric line shape with respect to the central wavelength. As in the case for the strong coupling, $\left|\alpha_{c}\right|$ reaches a maximum value between the two scattering peaks and close to the dip. In this case, the maximum value of $\left|\alpha_{c}\right|$ is 0.76 , which corresponds to $\lambda=655 \mathrm{~nm}$. Consequently, a system working in the moderate coupling regime provides better $\mathrm{CP}$ than a strongly coupled system.

The third configuration represents the weak coupling regime: the minor semiaxes $b$ of the elliptical dipole antennas are set to $30 \mathrm{~nm}$. Figure 1d shows the scattering cross section for this configuration with its asymmetric deformation with respect to the original mode around $\lambda=626 \mathrm{~nm}$ due to weak coupling. The charge distributions of the system at the resonance peak wavelengths $\lambda=610$ and $\lambda=647 \mathrm{~nm}$ and CP peak wavelength $\lambda=610 \mathrm{~nm}$ are shown in Figure $2 \mathrm{~d}-\mathrm{f}$ at different phases. The phase evolution of the charge distributions suggests that, at $\lambda=$ $647 \mathrm{~nm}$, the mode is more dipolar along the $x$-axis, which is also reflected in the amplitude of $\alpha_{\mathrm{c}}$ at those wavelengths. At $\lambda$ $=610 \mathrm{~nm}$, the mode is almost linear with tendency to be linearly polarized along the $x$-direction. At the overlap wavelength $\lambda=626 \mathrm{~nm}$, where the system shows a maximum for $\left|\alpha_{c}\right|$, the charges rotate in a roughly circular path. In fact at this wavelength, numerical calculations reveal that not only almost half of the energy absorbed by antenna (1) is coupled to antenna (2), but also, the two perpendicular dipoles radiate with a $\pi / 2$ phase difference, thus producing CP light. For the weak, intermediate and strong coupling cases, a fitting to the model introduced in ref 46 indicates that the position of maximum circular polarization does not correspond to the energy of any of the two underlying bright and dark modes but is rather located between them.

The magnitude of CP produced by weak coupling is similar to moderate coupling; nevertheless, the $40 \%$ higher scattering cross section, not shown here, is more suitable for far-field measurements. In the far-field, using an optical setup with limited numerical aperture, it is interesting to investigate to which extent the far-field pattern is circularly polarized. The angular distribution of $\alpha_{\mathrm{c}}$ for the weak coupling antenna at $\lambda=$ $626 \mathrm{~nm}$ is represented in Figure 3a. The surface extension and color indicate the magnitude and sign of $\alpha_{\mathrm{c}}$ as a function of the scattering direction. According to this distribution, the $+z$ hemisphere is right-handed, whereas the $-z$-hemisphere is lefthanded polarized, and furthermore, at $\varphi=0^{\circ}$ and $180^{\circ}$ and $\theta=$ $\pm 40^{\circ}$ (where $\theta=0^{\circ}$ corresponds to $z$-axis), the magnitude of $\mathrm{CP}$ approaches \pm 1 .

For our considerations the excitation was linearly polarized along the $x$-axis. For an excitation along the $y$-axis, as the structure is rotationally asymmetric, the optical response of the antenna exhibits a circular behavior as well, however, with opposite helicity than for the $x$-axis excitation. Figure $3 \mathrm{~b}$ and $\mathrm{c}$ displays the scattering cross section and the magnitude of $\alpha_{\mathrm{c}}$ with respect to the polarization of the excitation. At $\varphi=0^{\circ}, \varphi=$ 
$90^{\circ}$, and $\varphi=180^{\circ},\left|\alpha_{c}\right|$ reaches a maximum, whereas at $\varphi=45^{\circ}$ and $\varphi=135^{\circ} \alpha_{\mathrm{c}}$ drops to zero (Figure 3c), since for these last two polarizations the structure is symmetric with respect to the polarization axis.

With the help of the three model geometries investigated in this work, it has clearly been demonstrated that weak coupling generates higher CP conversion than strong coupling does. To further our understanding of the underlying mechanisms, a weakly coupled structure having a minor semiaxis $b=30 \mathrm{~nm}$, similar to that studied above, is investigated now. The major axis of the elliptical cavity in the overlap region is kept constant whereas its minor semiaxis is varied from close to zero to 25 $\mathrm{nm}$, where the cavity becomes circular. In Figure $4 \mathrm{a}$, the

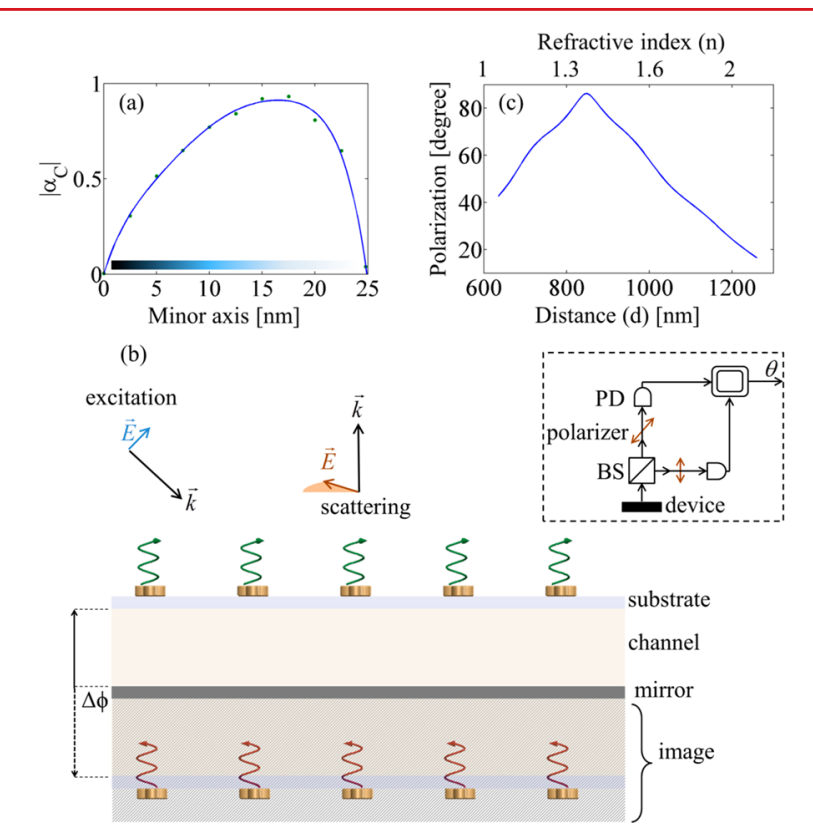

Figure 4. (a) Magnitude of $\left|\alpha_{c}\right|$ as a function of the length of the minor semiaxis of the elliptical perturbation. The horizontal color bar indicates the change in the perturbation strength, from highly perturbed system with a thin elliptical cavity (dark blue), to a nonperturbed system with a symmetric circular cavity (light blue). (b) Arrangement of an array of structures on a glass substrate at the top and a mirror at the bottom of a channel. The hachure part represents the image of the array mimicking a virtual array with opposite helicity. Inset: schematic illustration of the optical setup for the recognition of the output polarization. (c) Angle of the polarization of the resulting plane wave as a function of the distance $d$ between the layers (lower axis, refractive index is set to 1 ) and as a function of the change of the refractive index of the analyte in the channel when the height of the channel (structures to mirror) is fixed to $300 \mathrm{~nm}$.

maximum $\left|\alpha_{c}\right|$ is calculated and plotted as a function of the cavity minor axis. The horizontal bar in this figure indicates the perturbation strength. The darker part corresponds to narrower ellipses and the lighter part to wider ellipses, representing strong and weak perturbation. A maximum of $93 \%$ for the CP factor is achieved in the interval between weak and intermediate coupling.

Finally, as an application, we propose in this section a sensor based on the principle of generation of CP light using the plasmonic structures studied above. It is well-known that the superposition of two CPs with opposite helicity results in a linear polarization pointing in a direction which depends on the phase difference between the CPs. A device containing an array of antennas generating left-handed CP positioned on top and a mirror situated at the bottom of a channel is investigated. For the sake of clarity, a schematic illustration of the configuration is shown in Figure 4b. The mirror can be replaced by the image of the antennas situated at the top, forming a virtual array of antennas generating right-handed CP. The spacing distance $d$ between the actual plane and the image plane is either varied from about 600 to $1300 \mathrm{~nm}$ or filled with an analyte with a certain refractive index $n$ to be measured. The angle of the incident light is fixed at $\theta=45^{\circ}$, assuring a simultaneous illumination of both the bottom and top planes, causing the antennas in the two layers to radiate in two opposite helicities and with a constant phase difference. Consequently, the total response gives rise to a linear polarization in the far-field. For an observer in the $+z$ hemisphere, the orientation of the linear polarization depends on the phase shift caused by the phase difference originating from the distance $d$ of the two arrays and the refractive index $n$ of the material in the channel. The inset in Figure $4 b$ shows a schematic illustration of a setup for the detection of the polarization. The output of the device is divided into two paths using a beam splitter (BS) where the intensities of the light passing through two perpendicular polarizers placed in the two individual paths are measured using two photodiodes (PD). The polarization angle is finally obtained by calculating the ratio of the two PD-signals.

Figure $4 \mathrm{c}$ displays the change in the orientation of the linear polarization as a function of the change in the relative phase of the two waves stemming from the different plates, due to (1) change in the distance $d$ indicated by the bottom axis, while the refractive index is kept to constant to 1 or (2) change in the refractive index of the analyte in the channel indicated by the top axis, while the height of the channel is kept constant. The antennas are excited by a single wavelength plane wave with $\lambda=$ $626 \mathrm{~nm}$ in free space. The change in the orientation of the resulting linearly polarized light observed in the far-field is more than $45^{\circ}$ for a distance change $\Delta d$ of $200 \mathrm{~nm}$ or a change of the refractive index $n$ of 0.3 . Thus, the effective sensitivity is therefore better than $0.23 \mathrm{deg} \cdot \mathrm{nm}^{-1}$ or $33 \mathrm{deg} \cdot \mathrm{RIU}^{-1}$, respectively. Assuming an angular resolution of $0.01^{\circ}, 47$ a sensitivity of $33 \mathrm{deg} \cdot \mathrm{RIU}^{-1}$ means that the device is capable to resolve a change in the refractive index $n$ of $3 \times 10^{-4}$. For comparison: for a typical localized surface plasmon resonance (LSPR) sensor, a general sensitivity of $300 \mathrm{~nm} / \mathrm{RIU}$ can be assumed. ${ }^{48}$ Considering a measurement setup with the spectral resolution of $1 \mathrm{~nm}$, a change of the refractive index of $3.3 \times$ $10^{-3}$ can be diagnosed. This comparison suggests that the proposed sensor provides a high sensitivity. In contrast to LSPR sensors requiring a monochromator and camera, the setup proposed here is simpler and cheaper.

In summary, we have proposed a plasmonic structure composed of two perpendicular elliptical dipoles enabling the generation of circularly polarized light. When the structure is excited with linearly polarized light, a circularly polarized wave is reemitted into the far-field by virtue of an asymmetric aperture situated in the center of the structure. The asymmetry of this structure allows near-field coupling of the two perpendicular plasmonic modes along the $x$ - and $y$-axes with a phase shift of $90^{\circ}$, at a particular wavelength, giving rise to the emission of CP light. The plasmonic system at hand has been studies in terms of coupling strength revealing that the highest circular polarization amplitude occurs between the intermediate and weak coupling regimes. Finally, we have set the base for 
using this structure as a new platform for ultrasensitive low concentration analyte or liquid/gas pressure sensors.

\section{AUTHOR INFORMATION}

\section{Corresponding Author}

*E-mail: olivier.martin@epfl.ch.

\section{Notes}

The authors declare no competing financial interest.

\section{ACKNOWLEDGMENTS}

This work was supported by the Swiss National Science Foundation (NCCR Nanoscale Science, projects 200021_125326 and CR23I2_147279).

\section{REFERENCES}

(1) Petryayeva, E.; Krull, U. J. Anal. Chim. Acta 2011, 706 (1), 8-24.

(2) Atwater, H. A.; Polman, A. Nat. Mater. 2010, 9 (3), 205-213.

(3) Ozbay, E. Science 2006, 311 (5758), 189-193.

(4) Fischer, H.; Martin, O. J. F. Opt. Express 2008, 16 (12), 91449154.

(5) Alonso-González, P.; Albella, P.; Schnell, M.; Chen, J.; Huth, F.; García-Etxarri, A.; Casanova, F.; Golmar, F.; Arzubiaga, L.; Hueso, L. E.; Aizpurua, J.; Hillenbrand, R. Nat. Commun. 2012, 3, 684.

(6) Curto, A. G.; Volpe, G.; Taminiau, T. H.; Kreuzer, M. P.; Quidant, R.; van Hulst, N. F. Science 2010, 329 (5994), 930-933.

(7) Chang, W. S.; Lassiter, J. B.; Swanglap, P.; Sobhani, H.; Khatua, S.; Nordlander, P.; Halas, N. J.; Link, S. Nano Lett. 2012, 12 (9), 4977-4982.

(8) Hoflich, K.; Becker, M.; Leuchs, G.; Christiansen, S. Nanotechnology 2012, 23 (18), 185303.

(9) Papakostas, A.; Potts, A.; Bagnall, D. M.; Prosvirnin, S. L.; Coles, H. J.; Zheludev, N. I. Phys. Rev. Lett. 2003, 90 (10), 107404.

(10) Drezet, A.; Genet, C.; Laluet, J. Y.; Ebbesen, T. W. Opt. Express 2008, 16 (17), 12559-12570.

(11) Huang, L.; Chen, X.; Bai, B.; Tan, Q.; Jin, G.; Zentgraf, T.; Zhang, S. Light Sci. Appl. 2013, 2, e70.

(12) Yu, N. F.; Aieta, F.; Genevet, P.; Kats, M. A.; Gaburro, Z.; Capasso, F. Nano Lett. 2012, 12 (12), 6328-6333.

(13) Kuwata-Gonokami, M.; Saito, N.; Ino, Y.; Kauranen, M.; Jefimovs, K.; Vallius, T.; Turunen, J.; Svirko, Y. Phys. Rev. Lett. 2005, 95 (22), 227041.

(14) Drezet, A.; Genet, C.; Ebbesen, T. W. Phys. Rev. Lett. 2008, 101 (4), 043902.

(15) Li, T.; Liu, H.; Wang, S. M.; Yin, X. G.; Wang, F. M.; Zhu, S. N.; Zhang, X. A. Appl. Phys. Lett. 2008, 93 (2), 021110.

(16) Bachman, K. A.; Peltzer, J. J.; Flammer, P. D.; Furtak, T. E.; Collins, R. T.; Hollingsworth, R. E. Opt. Express 2012, 20 (2), 13081319.

(17) Ogut, E.; Sendur, K. Appl. Phys. Lett. 2010, 96 (14), 141104.

(18) Ginzburg, P.; Rodriguez-Fortuno, F. J.; Martinez, A.; Zayats, A. V. Nano Lett. 2012, 12 (12), 6309-6314.

(19) Gallinet, B.; Siegfried, T.; Sigg, H.; Nordlander, P.; Martin, O. J. F. Nano Lett. 2013, 13 (2), 497-503.

(20) Gallinet, B.; Martin, O. J. F. ACS Nano 2011, 5 (11), 89999008.

(21) Luk'yanchuk, B.; Zheludev, N. I.; Maier, S. A.; Halas, N. J.; Nordlander, P.; Giessen, H.; Chong, C. T. Nat. Mater. 2010, 9 (9), 707-715.

(22) Halas, N. J.; Lal, S.; Chang, W.-S.; Link, S.; Nordlander, P. Chem. Rev. 2011, 111 (6), 3913-3961.

(23) Sonnefraud, Y.; Verellen, N.; Sobhani, H.; Vandenbosch, G. A. E.; Moshchalkov, V. V.; Van Dorpe, P.; Nordlander, P.; Maier, S. A. ACS Nano 2010, 4 (3), 1664-1670.

(24) Fano, U. Phys. Rev. 1961, 124 (6), 1866.

(25) Kleinman, S. L.; Frontiera, R. R.; Henry, A. I.; Dieringer, J. A.; Van Duyne, R. P. Phys. Chem. Chem. Phys. 2013, 15 (1), 21-36.
(26) Fabris, L.; Schierhorn, M.; Moskovits, M.; Bazan, G. C. Small 2010, 6 (14), 1550-1557.

(27) Lovera, A.; Martin, O. J. F. SPIE Optics+Photonics 2011 Conference, San Diego, CA, Aug 20-25, 2011.

(28) Leverette, C. L.; Jacobs, S. A.; Shanmukh, S.; Chaney, S. B.; Dluhy, R. A.; Zhao, Y. P. Appl. Spectrosc. 2006, 60 (8), 906-913.

(29) Kats, M. A.; Genevet, P.; Aoust, G.; Yu, N. F.; Blanchard, R.; Aieta, F.; Gaburro, Z.; Capasso, F. Proc. Natl. Acad. Sci. U.S.A. 2012, 109 (31), 12364-12368.

(30) Yang, J.; Zhang, J. S. Plasmonics 2011, 6 (2), 251-254.

(31) Schaferling, M.; Yin, X. H.; Giessen, H. Opt. Express 2012, 20 (24), 26326-26336.

(32) Decker, M.; Zhao, R.; Soukoulis, C. M.; Linden, S.; Wegener, M. Opt. Lett. 2010, 35 (10), 1593-1595.

(33) Ginzburg, P.; Fortuno, F. J. R.; Wurtz, G. A.; Dickson, W.; Murphy, A.; Morgan, F.; Pollard, R. J.; Iorsh, I.; Atrashchenko, A.; Belov, P. A.; Kivshar, Y. S.; Nevet, A.; Ankonina, G.; Orenstein, M.; Zayats, A. V. Opt. Express 2013, 21 (12), 14907-17.

(34) Decker, M.; Klein, M. W.; Wegener, M.; Linden, S. Opt. Lett. 2007, 32 (7), 856-858.

(35) Kern, A. M.; Martin, O. J. F. J. Opt. Soc. Am. A 2009, 26 (4), $732-740$.

(36) Chuntonov, L.; Haran, G. Nano Lett. 2013, 13 (3), 1285-1290.

(37) Christofi, A.; Stefanou, N.; Gantzounis, G.; Papanikolaou, N. J. Phys. Chem. C 2012, 116 (31), 16674-16679.

(38) Biagioni, P.; Savoini, M.; Huang, J. S.; Duo, L.; Finazzi, M.; Hecht, B. Phys. Rev. B 2009, 80 (15), 153407.

(39) Fan, Z. Y.; Govorov, A. O. Nano Lett. 2010, 10 (7), 2580-2587.

(40) Liu, N.; Liu, H.; Zhu, S. N.; Giessen, H. Nat. Photonics 2009, 3 (3), 157-162.

(41) Biagioni, P.; Huang, J. S.; Duò, L.; Finazzi, M.; Hecht, B. Phys. Rev. Lett. 2009, 102 (25), 256801.

(42) Husakou, A.; Kelkensberg, F.; Herrmann, J.; Vrakking, M. J. J. Opt. Express 2011, 19 (25), 25346-25354.

(43) Valev, V. K.; Smisdom, N.; Silhanek, A. V.; De Clercq, B.; Gillijns, W.; Ameloot, M.; Moshchalkov, V. V.; Verbiest, T. Nano Lett. 2009, 9 (11), 3945-3948.

(44) Kern, A. M.; Meixner, A. J.; Martin, O. J. F. ACS Nano 2012, 6 (11), 9828-9836.

(45) Alu, A.; Engheta, N. Nat. Photonics 2008, 2 (5), 307-310.

(46) Gallinet, B.; Martin, O. J. F. Phys. Rev. B 2011, 83 (23), 235427.

(47) Matsubara, K.; Kawata, S.; Minami, S. Appl. Opt. 1988, 27 (6), 1160-1163.

(48) Stewart, M. E.; Anderton, C. R.; Thompson, L. B.; Maria, J.; Gray, S. K.; Rogers, J. A.; Nuzzo, R. G. Chem. Rev. 2008, 108 (2), 494-521. 